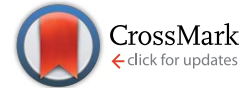

Cite this: J. Mater. Chem. C, 2015, 3, 2132

Received 25th November 2014

Accepted 6th January 2015

DOI: $10.1039 / c 4 t c 02693 d$

www.rsc.org/MaterialsC

\section{Inkjet printed paper based frequency selective surfaces and skin mounted RFID tags: the interrelation between silver nanoparticle ink, paper substrate and low temperature sintering technique $\uparrow$}

\author{
Veronica Sanchez-Romaguera, ${ }^{\text {*a }}$ Sebastian Wünscher, ${ }^{\text {bc }}$ Badredin M. Turki, ${ }^{d}$ \\ Robert Abbel, ${ }^{e}$ Silvia Barbosa, ${ }^{f}$ Daniel J. Tate, ${ }^{a}$ Dumtoochukwu Oyeka, ${ }^{d}$ \\ John C. Batchelor, ${ }^{d}$ Edward A. Parker, ${ }^{d}$ Ulrich S. Schubert ${ }^{\text {bc }}$ and Stephen G. Yeates ${ }^{\star a}$ \\ Inkjet printing of functional frequency selective surfaces (FSS) and radio frequency identification (RFID) tags \\ on commercial paper substrates using silver nanoparticle inks sintered using low temperature thermal, \\ plasma and photonic techniques is reported. Printed and sintered FSS devices demonstrate \\ performances which achieve wireless communication requirements having a forward transmission \\ scattering parameter, $S_{21}$, depth greater than $-20 \mathrm{~dB}$ at $13 \mathrm{GHz}$. Printed and plasma sintered RFID tags \\ on transfer paper, which are capable of being mounted on skin, improved read distances compared to \\ previously reported single layer transfer RFID tags fabricated by conventional thermal sintering.
}

\section{Introduction}

With an increasing dependence on wireless communication, the demand for the integration of electronic structures onto a broader range of substrates is becoming increasingly important. In this paper, we consider two such wireless structures, both of which have the unified requirement of having the need to print conductive features onto substrates which will not tolerate high processing temperatures and how consideration of the interplay between ink and paper substrate coupled with selection of the appropriate sintering techniques is essential in realizing optimum performance.

Firstly frequency selective surfaces (FSS) can be regarded as filters of electromagnetic waves and whilst well known in micro- and millimeter-wave aerospace systems, recent

\footnotetext{
${ }^{a}$ Organic Materials Innovation Centre (OMIC), School of Chemistry, The University of Manchester, M13 9PL, Manchester, UK. E-mail: veronica.sanchezromaguera@mbs.ac. uk; stephen.yeates@manchester.ac.uk

${ }^{b}$ Laboratory of Organic and Macromolecular Chemistry (IOMC), Friedrich Schiller University Jena, Humboldtstr. 10, 07743 Jena, Germany

'Jena Center for Soft Matter (JCSM), Friedrich Schiller University Jena, Philosophenweg 7, 07743 Jena, Germany

${ }^{d}$ School of Engineering and Digital Art, University of Kent, CT2 7NT, Kent, United Kingdom

${ }^{e}$ Holst Centre-TNO, High Tech Campus 31, 5656 AE Eindhoven, The Netherlands ${ }^{f}$ Condensed Matter Physics Department, Faculty of Physics, Universidade de Santiago de, Compostela, 15782 Santiago de Compostela, Spain

$\dagger$ Electronic supplementary information (ESI) available. See DOI: $10.1039 / \mathrm{c} 4$ tc02693d
}

applications in ultra-high frequency (UHF) mobile bands have given rise to new design and fabrication requirements, including large physical size and low-cost manufacture. ${ }^{\mathbf{1 , 2}}$ Current mobile and wireless systems are becoming severely capacity limited owing to interference and frequency reuse issues. ${ }^{3}$ A possible means to address the problem is to embed potentially large FSS panels within building structures such as FSS-wall paper, FSS-blinds, FSS-curtains and FSS-removable window covers. To be a realistic proposition, these panels must be simple to fabricate, low-cost and compatible with a wider range of materials, including temperature sensitive substrates. ${ }^{4}$ Aiming to address this gap, we recently reported the first digitally fabricated FSS on a temperature sensitive foil, polyethylene napthenate (PEN), which showed the potential of inkjet printing for the fabrication of FSS of similar performance to those fabricated with conventional etching techniques. ${ }^{5}$ Subsequent inkjet printed FSS reports have demonstrated both pre-coated textile and porous paper substrates $^{6}$ have necessitated multiple layer deposition (up to 10 layers) in combination with long sintering times (>60 min) and high sintering temperature $\left(160^{\circ} \mathrm{C}\right)$ in order to obtain sufficiently conductive features. ${ }^{7}$

Secondly passive radio frequency identification (RFID) tags which are used in both asset management and in monitoring, identifying and tracking people particularly in work environments such as power plants, airports, hospitals, military bases, refineries and access restricted areas is an emerging application area. Recently, we reported the inkjet fabrication of UHF RFID tags, which were transferable onto skin. Commercially available 
non-porous transfer tattoo paper, silver nanoparticle ink, and moderate temperature conventional thermal sintering $\left(135^{\circ} \mathrm{C}\right.$, $30 \mathrm{~min}$ ) were used to produce the UHF RFID tags. ${ }^{8}$ Although the potential of inkjet printing to obtain paper based electromagnetic structures such as FSS and UHF RFID has been demonstrated, the need for conventional thermal sintering techniques to convert the silver ink into suitably conductive features is still a limiting factor for applications on more thermally sensitive substrates and for roll-to-roll (R2R) manufacturing.

Alternative approaches to thermal sintering of silver nanoparticles have recently been reported enhancing the compatibility of the sintering step with thermally sensitive substrates and with R2R manufacturing capabilities. ${ }^{9}$ These have included tailoring the inks such that they can be processed at low temperatures ${ }^{10}$ the use of sintering additives, ${ }^{\mathbf{1 1}, 12}$ application of an electrical current, ${ }^{13}$ exposure to low- and atmospheric-pressure plasmas, ${ }^{\mathbf{1 4 , 1 5}}$ microwave irradiation ${ }^{\mathbf{1 6}}$ and several photonic approaches including infra-red (IR) ${ }^{17}$ laser $^{18}$ and intense pulsed light (IPL) sintering. ${ }^{19,20}$

Although some authors have reported sintering of silver nanoparticles on paper substrates ${ }^{21-24}$ and others have conducted feasibility studies on plastic substrates to compare the sintering effectiveness of various alternative lowtemperature sintering techniques, ${ }^{25}$ to the best of our knowledge, no comparative study on the interrelation between different silver nanoparticle inks, paper substrates and various alternative sintering methods in addition to conventional thermal sintering has been reported. This work provides a systematic study of the electrical performance of inkjet printed features by comparing two commercially available inks, two commercially available paper substrates and three low-temperature sintering techniques, thermal, low-pressure argon plasma and IPL with a focus on FSS and tattoo RFID applications. Conclusions are drawn with regards to combinations of ink-paper substrate-printing and sintering techniques with potential for R2R manufacturing and commercial viability.

\section{Experimental section}

\subsection{Materials}

Two commercial silver nanoparticle dispersions were used in this work. Ink A is a $20 \mathrm{wt} \%$ dispersion of silver nanoparticles, (particle diameter in the range of $50 \mathrm{~nm}$ by scanning electron microscopy (SEM)) in ethanol/ethylene glycol mixture from Sigma-Aldrich (SunTronic U5603 from Sun Chemicals). Ink B is a water-based dispersion from Clearjet Ltd. (Jerusalem, Israel) containing $30 \mathrm{wt} \%$ of silver nanoparticles having a diameter of $10 \mathrm{~nm}$. A detailed investigation of the composition of this ink can be found in a previously reported study. ${ }^{26}$

Two paper substrates engineered for inkjet printing were chosen. Inkjet tattoo transfer paper (tattoo paper), representative of the paper class having a polymeric receiving layer, was supplied by Crafty Computer Paper (Leicester, UK) whilst PEL Nano-P60 paper (PEL paper), representative of the paper class having an inorganic micro-porous receiving layer, was obtained from Printed Electronics Ltd. (Cambridge, UK). In all cases the substrates were purged with a flow of air to remove dust particles prior to use.

\subsection{Inkjet printing and sintering}

Inkjet printing was performed using a piezoelectric Dimatix DMP-2800 system (Dimatix-Fujifilm Inc., USA), equipped with a $10 \mathrm{pL}$ cartridge (DMC-11610). The cartridge temperature was set between 30 and $45{ }^{\circ} \mathrm{C}$ and was adapted to the applied substrate temperature.

Thermal sintering was carried out in a convection oven set at $150{ }^{\circ} \mathrm{C}$ unless otherwise stated. Sintering times of up to $60 \mathrm{~min}$ were used as stated in the main text. Plasma sintering was performed using a low-pressure argon plasma instrument from Diener Electronic (Nagold, Germany). Small samples were processed with the Diener pico plasma chamber operating at a power of $190 \mathrm{~W}$ and a processing pressure of 0.4 mbar. Large area samples (A4 FSS) were processed with the Diener nano plasma chamber operating at a power of $300 \mathrm{~W}$ and a pressure of 0.4 mbar. Photonic flash sintering was carried out with a custom tool previously described. ${ }^{27,28}$ It consists of a combination of a XOP-15 lamp in an elliptical reflector and a commercially available flash lamp system (Sinteron 2000 from Xenon Corp., U.S.A.) mounted above a substrate holder which can be transported underneath the lamps at a predefined speed up to $20 \mathrm{~m} \mathrm{~min}^{-1}$.

\subsection{Characterization}

Thermogravimetric analysis (TGA) was performed under ambient atmosphere in the range from room temperature to $600{ }^{\circ} \mathrm{C}$ at a heating rate of $10{ }^{\circ} \mathrm{C} \mathrm{min}^{-1} \mathrm{using}$ a TGAQ5000 system from TA Instruments (NewCastle, USA). The electrical resistance of sintered silver features was measured by the 4point probe technique. A Jandel multi-position wafer probe system (Jandel Engineering Ltd., Leighton Buzzard, UK) mounted with a cylindrical probe head (solid tungsten carbide needles of $0.40 \mathrm{~mm}$ diameter spaced $1.0 \mathrm{~mm}$ ) was employed. Grazing angle FT-IR experiments were performed on a Vertex-70 spectrometer equipped with a Harrick Seagull accessory and Germanium hemisphere ATR crystal and nitrogen purge from Bruker. The sample was mounted with an incident angle of $65^{\circ}$. Background and measurements were averaged over 64 scans with a resolution of $4 \mathrm{~cm}^{-1}$, recorded by a DLaTGS detector.

Optical microscopy images were taken using a Leica ${ }^{\circledR}$ DM25000 M optical microscope with adjustable magnification in reflective mode. Scanning electron microscopy (SEM) images were taken using an EVO® LS 15 system (Carl Zeiss AG, Oberkochen, Germany) operating at an accelerating voltage of $20 \mathrm{kV}$.

The FSS design used in this work has been described in our earlier work ${ }^{30}$ consisting of 374 patch dipoles set on a skewed lattice (Fig. S7†). The RFID antenna used in this work has a total size of $65 \times 20 \mathrm{~mm}^{2}$ including a port area, and is described in detail in our previous study (Fig. S7†). ${ }^{9}$ Tag read distance was measured using a Voyantic Tagformance lite RFID measurement system. After calibrating the system at $35 \mathrm{~cm}$ the RFID tag was transferred to the volunteers arm and the read range was 
extrapolated by measurement at the global RFID UHF frequency bands within permitted transmission power levels.

\section{Results and discussion}

\subsection{Characterization of paper substrates}

The papers used in this study are representative of the two major classes of commercial inkjet paper, polymer coated and inorganic micro-porous. The composition and thermal stability of polymer coated tattoo transfer paper has previously been described and comprises a $1 \mu \mathrm{m}$ thick non-porous ink-receiving layer primarily composed of polyvinyl alcohol (PVA) which limits the sintering temperature to $135{ }^{\circ} \mathrm{C}$ (Fig. S1a-c $\dagger$ ). ${ }^{8}$ By contrast, PEL paper, specifically designed for printed electronic applications, comprises a inorganic micro porous ink-receiving layer with a porosity of $60 \mathrm{~nm}$ which can withstand a sintering temperature of up to $150{ }^{\circ} \mathrm{C}^{30}$ Analysis of the PEL paper by scanning electron microscopy (SEM), Fourier transform infrared spectroscopy (FTIR) and thermogravimetric analysis (TGA) (Fig. S1d-f $\dagger$ ) clearly shows two layers: a top layer of approximately $20 \mu \mathrm{m}$ thickness, confirmed by FTIR to be silica based micro porous inorganic ink-receiving layer and a bottom paper base layer. The inorganic nature and the greater thickness of PEL paper ink-receiving layer are attributed to its greater thermal stability.

\subsection{Ink/substrate interactions}

Printing of defect free features is a key requirement to manufacture highly conductive components which are suitable as antennas for RFID tags and conductive arrays in FSS applications. In order to identify appropriate print conditions for the fabrication of such structures, variables including drop-to-drop distance (drop spacing) and substrate temperature were studied for the different ink/paper combinations.

For Ink A (ethanol/ethylene glycol based dispersion), printing simple single layer patterns with a drop spacing ranging between 15 and $25 \mu \mathrm{m}$ on both paper substrates was found to result in well-defined patterns without any signs of bulging or ill-defined edges. However, single layer patterns printed with a drop spacing of $25 \mu \mathrm{m}$ on PEL paper occasionally showed isolated defects resulting from non-overlapping drops of successively printed adjacent lines. The reason for this effect is the fast drying of the deposited ink due to the micro porous ink-receiving layer which allows fast removal of the solvent.

The reduction of the resistance of a printed conductor is accessible by increasing the pattern height via multilayer printing. Whilst double and triple layer printing was possible on PEL paper, severe defects due to drying effects were observed for Ink A on tattoo paper. It is believed that the main reason for these defects is the relief of mechanical stress resulting from a slow drying of the ink (mud cracking). ${ }^{31}$ In order to accelerate drying on the tattoo paper, the substrate temperature was increased from $32{ }^{\circ} \mathrm{C}$ to up to $60{ }^{\circ} \mathrm{C}$ and combined with a reduction of deposited material during each layer by increasing the drop spacing, resulting in defect free patterns of up to three layers. These substrate temperatures are, however, a trade-off against print stability and reliability since they also accelerate solvent evaporation at the print head and, therefore, cause frequent nozzle clogging.

Printing of Ink B (water-based dispersion) on PEL paper is achievable by using similar settings as in the case of Ink A (15 to $25 \mu \mathrm{m}$ drop spacing, $32{ }^{\circ} \mathrm{C}$ substrate temperature). When printing onto tattoo paper, Ink B shows strong wetting of the substrate resulting in ill-defined pattern dimensions. Suitable edge definition was achieved by adjusting the drop spacing from 40 to $50 \mu \mathrm{m}$ and the substrate temperature to $50^{\circ} \mathrm{C}$, head temperature adapted to $40{ }^{\circ} \mathrm{C}$. However the printed films possess high surface roughness, which is disadvantageous for both, high electrical conductivity and printing of multiple layers. As a consequence multilayer printing was not achievable with sufficient quality in terms of wetting and surface homogeneity. This study shows the importance of matching ink fluid properties to the engineered properties of the substrate and how careful consideration of print conditions can be used to optimize the feature quality.

\subsection{Sintering studies}

In order to study the potential of low-pressure argon plasma and IPL low temperature sintering on tattoo and PEL paper comparison is first made to conventional thermal sintering. Based on the thermal stability analysis, conventional thermal sintering temperature was limited to $135^{\circ} \mathrm{C}$ for tattoo paper and $150{ }^{\circ} \mathrm{C}$ for PEL paper.

3.3.1. Thermal sintering. Thermal sintering of Ink A on tattoo paper has previously been reported showing best electrical properties were achieved sintering for $30 \mathrm{~min}$ (Fig. 1). ${ }^{8}$ In order to evaluate the electrical properties of Ink A on PEL paper, sheet resistances of samples printed with a drop spacing of 15 , 20 and $25 \mu \mathrm{m}$ were determined for thermal sintering of up to $30 \mathrm{~min}$. Obtained sheet resistances are dependent on the

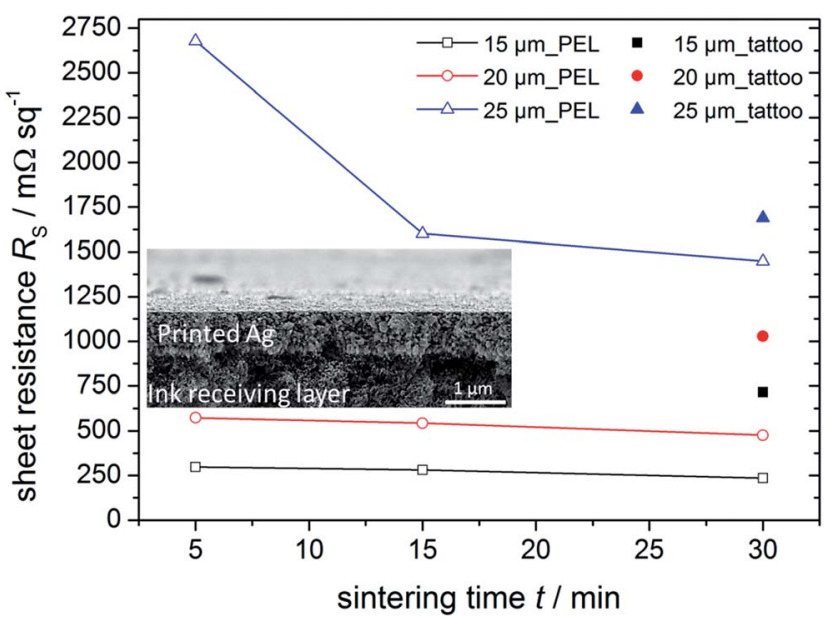

Fig. 1 Sheet resistance of Ink A on PEL paper as function of sintering time for thermal sintering at $150{ }^{\circ} \mathrm{C}$ (open icons and solid lines) and sheet resistance of Ink $A$ on tattoo paper after 30 min of thermal sintering at $135{ }^{\circ} \mathrm{C}$. Cross-sectional SEM image of thermally sintered Ink A pattern (dot spacing $20 \mu \mathrm{m}$ ) on PEL paper (inset). 
amount of deposited material i.e. higher amount of deposited material results in lower sheet resistances. Using an $15 \mu \mathrm{m}$ drop spacing and a sintering temperature of $150{ }^{\circ} \mathrm{C}$ for 5 to $30 \mathrm{~min}$, sheet resistances as low as 300 and $235 \mathrm{~m} \Omega \mathrm{sq}^{-1}, 5 \times$ resistivity of bulk silver, ${ }^{8}$ were reached. In comparison to the thermal sintering results on tattoo paper, these values are significantly better due to the combination of slightly higher sintering temperature, higher thermo-mechanical stability of the inkreceiving layer, improved removal of solvents as well as favorable sintering effects due to the silica containing surface of the PEL paper. An increase in sheet resistance at longer sintering times due to damage of the substrate was not observed as in the case of tattoo paper, where prolonged heating leads to a softening and wrinkling of the polymeric receiving layer and, therefore, to macroscopic damage of the printed silver layer (Fig. S2 and S3 $\dagger$ ). ${ }^{8}$ Following the trend of lower resistance for increased pattern height, 3 layer printing reduced the sheet resistance further to $92 \mathrm{~m} \Omega \mathrm{sq}^{-1} 15 \mu \mathrm{m}$ drop spacing.

Previous studies on Ink B have shown that good conductivity can be achieved when sintered at $150{ }^{\circ} \mathrm{C}$ for at least $30 \mathrm{~min}$ on glass. ${ }^{32}$ Sintering on tattoo paper at both $135{ }^{\circ} \mathrm{C}$ and $150{ }^{\circ} \mathrm{C}$, whilst accepting substrate damage, for up to $60 \mathrm{~min}$, however, gave no measurable resistance. SEM images (Fig. 2b) did not show any signs of grain coarsening but particle sizes comparable to the as-printed ink. Whilst high surface roughness and a significantly lower amount of deposited ink per layer could be reasons for the comparatively high resistance, the absence of any sintering can only be due to the paper used here. The impact of the thermal conductivity of the substrate material, for instance, is known to have a large impact on the sintering result of silver nanoparticle inks. ${ }^{33}$ As indicated in the previously reported resistivity model for this particular ink, prolonged sintering times can lead to conductive patterns at lower temperatures. ${ }^{32}$ From a processing as well as substrate damage point of view, sintering times in the range of several hours at these temperatures are, however, not compatible with low cost and high throughput production.

In contrast to the unsuccessful sintering on tattoo paper, thermal sintering of Ink B on PEL paper using the same conditions reveals excellent sheet resistances of 174, 590 and
$940 \mathrm{~m} \Omega \mathrm{sq}^{-1}$ after $30 \mathrm{~min}$ for single layer patterns printed with drop spacing of 15, 20 and $25 \mu \mathrm{m}$, respectively, with double layer printing showing a further significant resistance reduction (Fig. 2a). All samples show a significant drop in sheet resistance within the first 15 min of sintering and significant densification and grain coarsening of the nanoparticles being observed by SEM (Fig. 2c) compared with un-sintered silver nanoparticle ink. ${ }^{28}$ It has to be stated that despite any deformation of the PEL paper, significant yellowing is observed for sintering times above $15 \mathrm{~min}$, indicating a slow decomposition of substrate components.

These results of Ink B on PEL and tattoo paper, in particular the differences of the SEM images, clearly show the influence of different substrate materials on the sintering performance. The silica based ink receiving layer of PEL paper is believed to have beneficial effect on the thermal sintering of nanoparticle based inks. Allen et al. reported on the sintering of a silver nanoparticle ink at room temperature and high humidity resulting in conductive patterns after one day. ${ }^{34}$ It is believed that these effects are due to synergistic enhanced water uptake by the silanol groups and a high bonding affinity of the stabilizer to the silica surface. The results for thermal sintering of Ink A and Ink B on PEL paper also reveal favorable effects in comparison to sintering on tattoo paper or on glass substrates, even without any humidity treatment.

3.3.2 Low-pressure argon plasma sintering. Previous studies regarding plasma sintering of Ink A on polymer foils show its ability to be sintered via plasma irradiation to highly conductive patterns. ${ }^{35,36}$ During plasma sintering (Diener pico) of Ink A on tattoo paper, however, severe cracking of the printed pattern was observed, which resulted in highly resistive or even non-conductive patterns (Fig. 3c). Cross-sectional SEM images of plasma sintered Ink A on tattoo paper reveal that the nanoparticles are successfully sintered and that macroscopic defects are the cause of the absence of measurable conductivity (Fig. 3b). It was found that the magnitude of cracking correlated to both the amount of deposited material, with multilayer patterns showing more pronounced cracking than single layer and whether the pattern was pre-dried. Since the top layers are sintered preferentially, evaporation of large amounts of
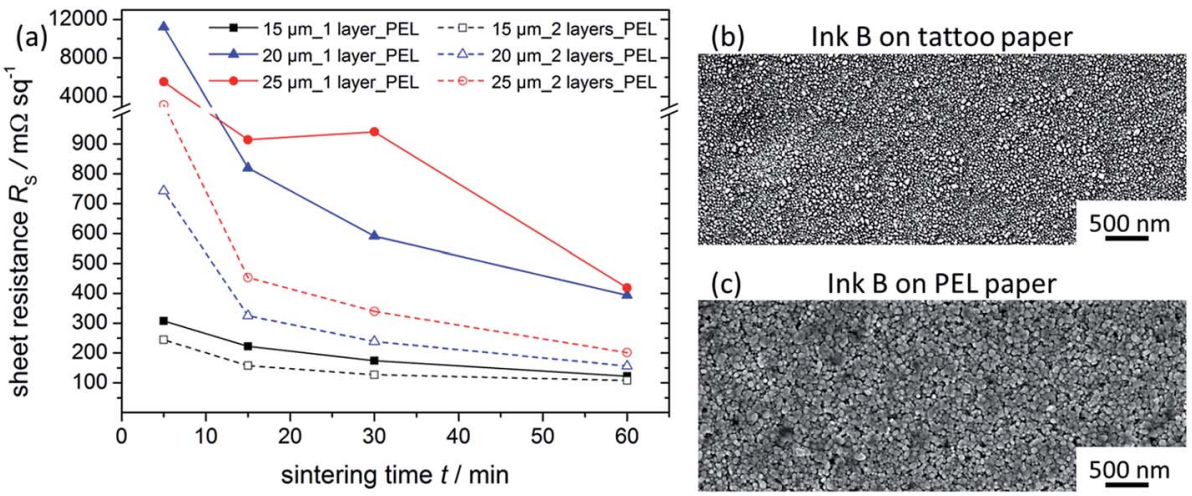

Fig. 2 Sheet resistance of single (solid lines and icons) and double layer (dashed lines and open icons) patterns of Ink B on PEL paper as function of sintering time for thermal sintering at $150{ }^{\circ} \mathrm{C}$ (a), SEM images of thermally sintered patterns of Ink B on tattoo (b) and PEL (c) paper. 

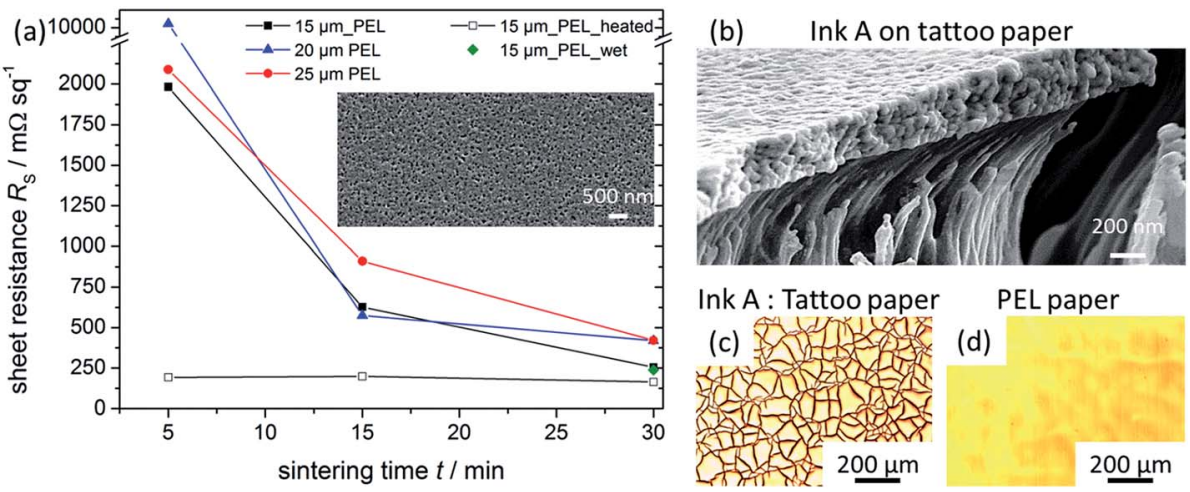

Fig. 3 Sheet resistances of low-pressure argon plasma sintered single layer patterns of Ink $\mathrm{A}$ on PEL paper after pre-drying at $100{ }^{\circ} \mathrm{C}$ for 5 min (solid lines and icons), without pre-drying (green diamond data point) and in combination with a pre-heated ground platen of the plasma device (Diener pico) (a). SEM image of Ink A on top of the ink receiving layer of tattoo paper (b), microscopy images of Ink A on tattoo (c) and PEL paper after 30 min of plasma sintering (d).

retained solvent from within the pattern can break the already formed crust layer, resulting in irreversible defects. Sintering of multilayer patterns of Ink A on tattoo paper using the larger Diener nano plasma device was found to yield defect free films with sheet resistances of $300 \mathrm{~m} \Omega \mathrm{sq}^{-1}$ (Fig. S5†) supporting previous observations on the sensitivity to electrode architecture. $^{33}$ The less stringent conditions in the larger plasma chamber enable milder sintering at lower substrate temperatures, slow heat dissipation due to plasma, which avoids damage to both the thermo-sensitive ink receiving layer of the tattoo paper as well as the sintered metal pattern. In comparison to sintering on tattoo paper, plasma sintering of Ink A on PEL paper results in patterns with less defects and consequently significantly reduced sheet resistances with triple layer printing possible if the pattern was pre-dried $\left(5 \mathrm{~min}\right.$ at $\left.100{ }^{\circ} \mathrm{C}\right)$ to minimize cracking defects and therefore enhance the electrical performance to sheet resistances from 250 to $110 \mathrm{~m} \Omega \mathrm{sq}^{-1}$ for single and triple layer patterns respectively at $15 \mu \mathrm{m}$ dot spacing (Fig. 3a and d). The beneficial influence of substrate heating during plasma sintering was enhanced by pre-heating of the substrate holder of the plasma chamber, which resulted in improved sheet resistances after only 5 min of sintering by one order of magnitude without causing damage to the substrate or the metal pattern (Fig. 3a). ${ }^{36}$

The results of plasma sintering of Ink B on both paper substrates are displayed in Fig. 4a. Sintered patterns did not show any signs of crack formation after sintering. Since Ink B is aqueous based, unlike Ink A which is glycol based, residual solvents can be more effectively removed from the as-printed pattern either during a pre-drying stage or during printing via elevated substrate temperatures. Due to the very high surface roughness of the printed patterns on tattoo paper, reliable sheet resistance values could not be measured and consequently the resistance values of an $8 \times 0.4 \mathrm{~mm}^{2}$ line are reported in Fig. 4 . For Ink B, a significant decrease in resistance is observed on both, tattoo and PEL paper, down to approximately 11.6 and $3.3 \Omega$, respectively. For comparison to other sintering techniques in this study, sheet resistance values of samples after 30 min of plasma sintering are provided in Fig. 4d. Plasma sintering of Ink B results in comparable values to plasma

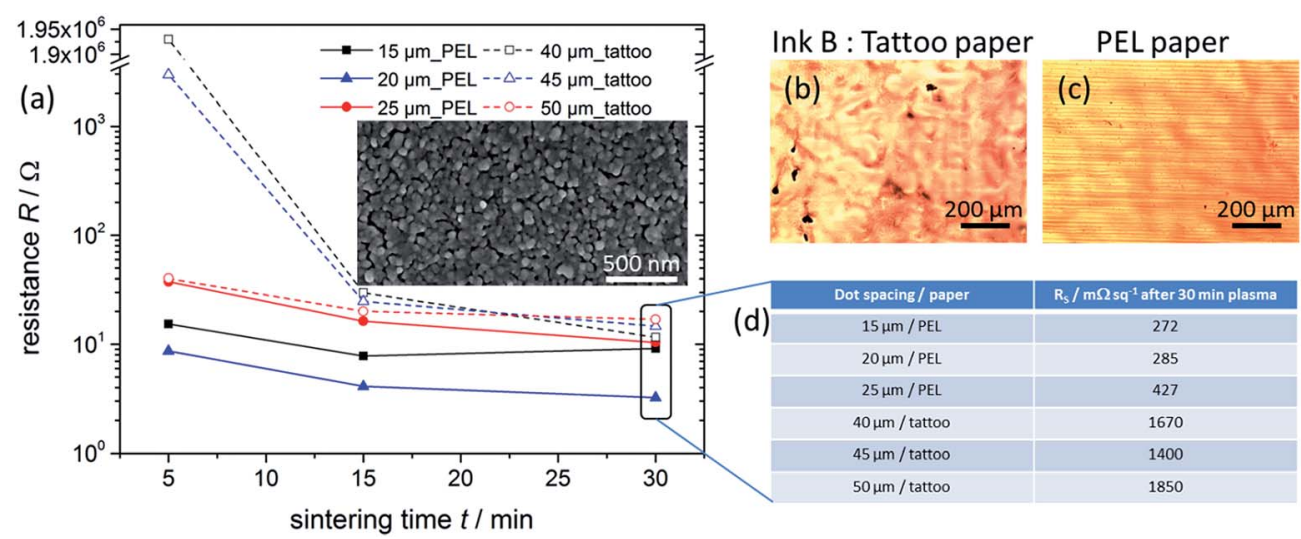

Fig. 4 Resistances of low-pressure argon plasma sintered single layer patterns of Ink B on PEL after pre-drying (solid lines and icons) and on tattoo paper without additional pre-drying (dashed lines and open icons) (a). SEM image of plasma sintered Ink B (both papers) (a, inset). Microscopy images of plasma sintered patterns of Ink B on tattoo (b) and PEL (c) paper, corresponding sheet resistance values after 30 min of plasma sintering (Diener pico) (d). 
sintering of Ink A when sintered on PEL paper. Despite its rather high surface roughness on tattoo paper, Ink B shows no signs of crack formation during plasma sintering and yields improved resistance values compared to thermal sintering on tattoo as well as PEL paper.

Unlike thermal sintering, lower sheet resistances upon increasing amount of deposited material was generally not observed for plasma sintering (Fig. 3a and 4a). This is because plasma sintering progresses top down with thicker layers requiring longer times for complete sintering throughout the entire layer. In combination with a heated substrate, this can be partially off-set although it is highly ink and substrate dependent as can be seen from the comparison of Fig. $3 \mathrm{a}$ and $4 \mathrm{a}^{\mathbf{1 4 , 1 5}}$

3.3.3 Intense pulsed light (IPL) sintering. IPL has been reported earlier as a fast and efficient processing method to achieve highly conductive patterns from metal based inks printed onto temperature sensitive substrates. ${ }^{33}$ Due to its selective and thus spatially localized heating mechanism, this can significantly reduce the thermal stress and, thus, damage to the substrate. Whereas there are numerous reports on this technique for polymeric foils, only a limited number of studies have been published on its use on paper. A study of photonic sintering on a variety of commercially available paper substrates of an ink having identical characteristics to Ink A resulted in highly conductive features. ${ }^{21}$ The sintering details, however, are not provided making direct correlation impossible. IPL sintering studies using Ink B are neither published on paper nor on polymer or inorganic substrates. Within this study, IPL sintering has been applied to samples of Inks A and B on both paper substrates in order to identify conditions which will produce features of sufficient electrical performance to be used for UHF RFID and FSS applications studied in this contribution.

IPL sintering of single layer Ink A on PEL paper resulted in homogeneously sintered films showing successful grain coarsening and coalescence of the silver nanoparticles by SEM with few micro cracks mainly resulting from residual solvent in the printed ink (Fig. S4 $\dagger$ ), which are further reduced by predrying. The effect of increasing pulse energies on the resistance of Ink A on PEL paper at a belt speed of $34 \mathrm{~mm} \mathrm{~s}^{-1}$ is shown in Fig. 5a. The optimal window of operation is rather narrow, 900 to $1100 \mathrm{~J}$ per pulse, since at lower values no significant improvement in sheet resistance is observed, whereas higher energies increase the risk of partial ablation of the silver from the substrate, resulting in non-functional structures. For multilayer patterns, a decrease in sheet resistance with increasing number of layers is observed. Whilst in comparison to thermal and plasma sintering, IPL sintering yields slightly higher sheet resistance, the processing time is reduced by several orders of magnitude (milliseconds) demonstrating a significant step towards high-throughput production compatibility.

IPL sintering of single layer Ink A on tattoo paper gave similar results to PEL paper. However multi-layered patterns suffered severe cracking, irrespective of pre-drying, as a result of the deformation of the low $T_{\mathrm{g}}$ ink receiving layer due to an excess of heat being transferred into the substrate resulting in localized softening. Excess energy is dissipated into the top layers of the substrate material, which permanently warps, if too much energy is used during IPL sintering. ${ }^{37,38}$ Since the ink receiving layer of the tattoo paper is very thin, this particular substrate does not tolerate heat conduction of this magnitude. These findings are analogous to using too high temperatures in an oven, hence, it is believed that the poor electrical performance of multilayer structures is due to substrate damage as detailed in our previous work. ${ }^{8}$ This conclusion is further supported by microscopy images of defective patterns revealing undulated features, which are similar to the defects of the substrate observed after excessive thermal sintering (Fig. S2 and s6a $\dagger$ ). Further optimization could potentially improve the performance of multilayer patterns.

Within this study, it was not possible to find settings to successfully sinter Ink B using IPL sintering on either of the paper substrates without significant damage (Fig. S6b and $c^{\dagger}$ ). As seen from the thermal sintering results Ink B requires significantly more energy input to be successfully sintered compared to Ink A supported by prior reports on polymer foil and glass. ${ }^{15,32,35}$ In order to achieve sintering of Ink B using IPL sintering, more energy has to be coupled into the ink, which

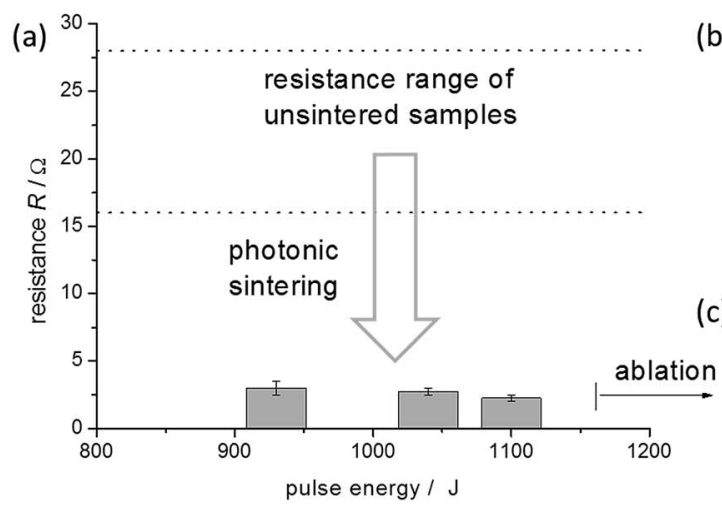

(b)

\begin{tabular}{|c|c|c|c|}
\hline Paper & Layers & $R_{s} / m \Omega s q^{-1}$ & $R_{\text {line }} / \Omega$ \\
\hline tattoo & 1 & 1,975 & 11 \\
\hline tattoo & 2 & $>20 \times 10^{6}$ & $5.2 \times 10^{6}$ \\
\hline tattoo & 3 & $>20 \times 10^{6}$ & $>20 \times 10^{6}$ \\
\hline PEL & 1 & 1,642 & 10 \\
\hline PEL & 2 & 695 & 5 \\
\hline PEL & 3 & 464 & 3 \\
\hline \multicolumn{4}{|c|}{ 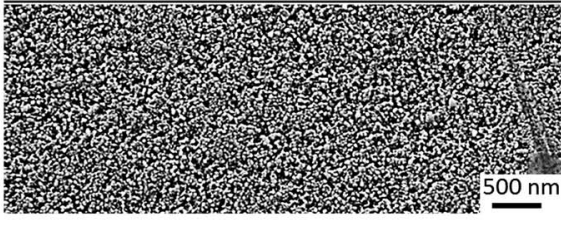 } \\
\hline
\end{tabular}

Fig. 5 Influence of varying pulse energies on the resistance of triple layer patterns of Ink A on PEL paper at a substrate speed of 34 mm s ${ }^{-1}$, a pulse frequency of $1.8 \mathrm{~Hz}$ and a pulse length of $2.0 \mathrm{~ms}$ (a). Sheet resistances of photonic sintered patterns of Ink A on tattoo and PEL paper at 20 $\mu \mathrm{m}$ dot spacing (b). SEM image of Ink A on PEL after photonic sintering (c). 
directly translates into a higher temperature as well as enhanced heat dissipation into the substrate material.

\subsection{UHF electromagnetic devices}

3.4.1 Frequency selective surfaces. To demonstrate the applicability of the various sintering techniques discussed in this paper, A4 size FSS structures (Fig. S7 $\dagger$ ) on paper substrates were fabricated using optimized printing and sintering conditions described earlier. The FSS structures presented in this paper are designed to block signals around $13 \mathrm{GHz}$ to a depth of greater than $-20 \mathrm{~dB}$ which is taken as the limit of acceptable performance which would be suitable for real-life applications, corresponding to $1 \%$ signal transmission through structure. ${ }^{29}$ Fig. 6 shows the measured FSS transmission response, $S_{21}$, of thermal, photonic and plasma sintered single layer of Ink A on tattoo and PEL paper.

In comparison to small scale samples, homogeneous sintering of Ink A on tattoo paper by plasma and IPL at A4 scale proved to be difficult and yielding dipoles with many defects due to crack formation and damage to the substrate resulting in comparatively high resistances, and as a consequence, $S_{21}$ were insufficient and in the range of $-11 \mathrm{~dB}$. This is in contrast to thermally sintered structures which gave $S_{21}$ of $-23 \mathrm{~dB}$ at about $13 \mathrm{GHz}$. Single layer plasma, IPL and thermally sintered FSS on PEL paper, however, all gave $S_{21}$ of -21 to $-24 \mathrm{~dB}$ at $15 \mu \mathrm{m}$ drop

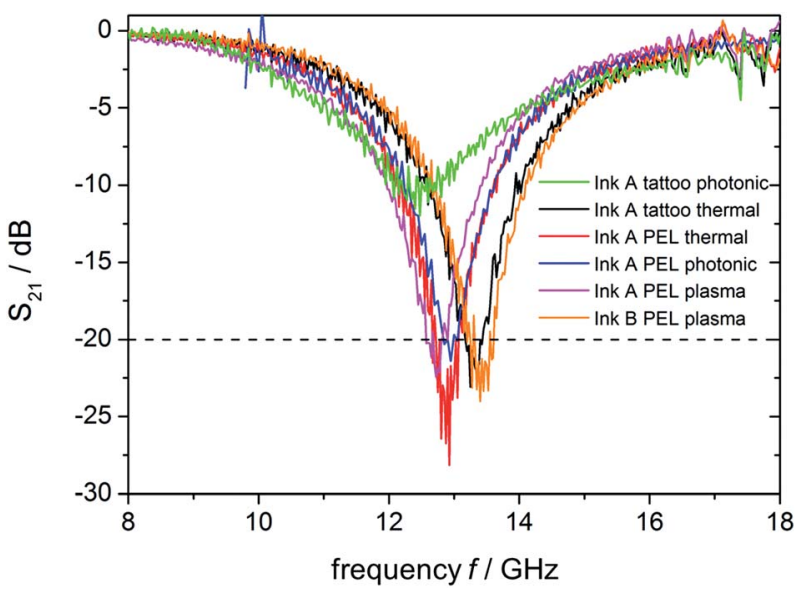

Fig. 6 Measured transmission response $\left(S_{21}\right)$ of single layer FSS fabricated with Ink $A$ on tattoo and PEL paper sintered by thermal, photonic and plasma means and Ink B on PEL paper sintered by plasma means. spacing and high reflectivity of the surface at about $13 \mathrm{GHz}$. The transmission response could be further improved from -24 to $-27 \mathrm{~dB}$ by increasing the number of deposited layers from one to three. Differences in transmission response values are attributed to differences in the electrical performance of printed dipoles achieved for each paper/sintering technique combination as detailed earlier (Table S1 $\dagger$ ). These transmission response values are comparable to those reported for the same FSS structure inkjet printed on PEN foil with a similar ink thermally sintered at $160{ }^{\circ} \mathrm{C}$ for $120 \mathrm{~min}$ (ref. 5) and comparable to copper wet-etched FSS (Table S1†) with the slightly different resonant frequencies a consequence of slightly different dipole lengths in the two cases. ${ }^{5}$

For Ink B, only FSS structures on PEL paper sintered via thermal and plasma sintering were considered here since a low surface roughness of the printed pattern could not be achieved by printing Ink B on tattoo paper. Whilst small area thermal sintering of Ink B on PEL paper yielded printed features of good electrical performance, $2.3 \Omega$ per dipole, A4 FSS produced using the same optimized printing and thermal sintering procedure had a transmission response of only $-11 \mathrm{~dB}$, since the dipole resistances were now between 70 to $160 \Omega$ per dipole. These adverse upscaling effects of thermal sintering are believed to be due to the higher relative volume of paper acting as a large heat sink and therefore retarding the sintering process. This effect is more pronounced in the case of Ink B because the temperatures required for sintering are significantly higher compared to Ink A.

Since plasma sintering is not based on thermal effects, heat sink effects of up-scaled are not as prevalent. Plasma sintered FSS fabricated with Ink $\mathrm{B}$ yielded transmission responses of $-23 \mathrm{~dB}$, which is comparable to those obtained for Ink A, Fig. 6 . This is an encouraging result which demonstrates that functional electromagnetic devices of similar performance are not limited to simply one ink. Sintering, however, has to be adapted according to the used ink/substrate combination.

3.4.2 Tattoo RFID tags. For the purpose of comparability to previously reported results, tag design, Fig. S7c, $\dagger$ and measurement setup, materials (Ink A) on tattoo paper, remained constant.

Single layer antennas were printed on tattoo paper, pre-dried at $70{ }^{\circ} \mathrm{C}$ and subsequently processed via low-pressure argon plasma (Diener nano, $30 \mathrm{~min}$ ) and IPL sintering. After application of the integrated circuit (IC) chip to the port area of the antenna, the tag was transferred to the forearm of a volunteer

Table 1 Summary results of RFID tags manufactured by inkjet printing comparing with thermal sintering, ${ }^{8}$ plasma and IPL sintering for Ink A

\begin{tabular}{|c|c|c|c|c|c|c|}
\hline Material & Fabrication & Sintering & Ink layers & $R_{\mathrm{p} 2 \mathrm{p}} / \Omega$ & Read distance ${ }^{a} / \mathrm{cm}$ & Ref. \\
\hline Ink A & Inkjet & Plasma & 1 full antenna & 17 & 46 & - \\
\hline Ink A & Inkjet & IPL & 1 full antenna & 12 & 50 & - \\
\hline Ink A & Inkjet & Thermal & 1 full antenna & 116 & 12 & 9 \\
\hline Silver paste & Stencil & N/A & 1 full antenna & 27 & 45 & 39 \\
\hline $\mathrm{Cu}$ metal & Etching & N/A & 1 full antenna & 0.17 & 75 & 39 \\
\hline
\end{tabular}

${ }^{a}$ Within designated UHF RFID bands: 865 to $868 \mathrm{MHz}$ for Europe and 902 to $928 \mathrm{MHz}$ for North America. 
Table 2 Overview of selected sintering results of different inks, papers and sintering techniques

\begin{tabular}{|c|c|c|c|c|c|c|c|c|c|c|c|c|}
\hline Substrate & \multicolumn{4}{|c|}{ Thermal sintering } & \multicolumn{4}{|c|}{ Plasma sintering } & \multicolumn{4}{|c|}{ IPL sintering } \\
\hline Successful sintering & $\checkmark$ & $x$ & $\checkmark$ & $\checkmark$ & $\checkmark$ & $\checkmark$ & $\checkmark$ & $\checkmark$ & $\checkmark$ & $x$ & $\checkmark$ & $x$ \\
\hline Timescale/min & 30 & - & $<30$ & 5 & 30 & 15 & 15 & 5 & $<1 \mathrm{~s}$ & - & $<1 \mathrm{~s}$ & - \\
\hline Min. $R_{\mathrm{sq}} / \mathrm{m} \Omega \mathrm{sq}^{-1}$ & 716 & - & 236 & 174 & 300 & $15 \Omega$ & 255 & $3 \Omega$ & 1975 & - & 464 & - \\
\hline
\end{tabular}

and investigated regarding read range at different frequencies. The functionality of the RFID tags relies on high conductivity of the antenna system, particularly on the feed track of the port area. Therefore, a low port-to-port resistance $\left(R_{\mathrm{p} 2 \mathrm{p}}\right)$ is desired and taken as reference resistance in this study. As shown in Table 1, antennas fabricated by plasma and IPL sintering methods resulted in UHF RFID tags of substantially superior read distance, 46 and $50 \mathrm{~cm}$ initial read range respectively, to those fabricated by thermal sintering $12 \mathrm{~cm}$ and of equal or superior performance to those fabricated using stencil applied silver paste $45 \mathrm{~cm}$. This is attributed to the comparatively improved electrical properties, $R_{\mathrm{p} 2 \mathrm{p}}$, of the antennas, whilst the lower $R_{\mathrm{p} 2 \mathrm{p}}$ and higher read distance of the copper etched tag, 75 $\mathrm{cm}$, is in part due to its greater layer thickness $(\geq 18 \mu \mathrm{m})$ in comparison to the inkjet printed antennas $(<1 \mu \mathrm{m})$. Although multilayer printing can lead to improved electrical performance and, hence, improved read range, this has an associated cost penalty. Plasma and especially photonic sintering, which is a process that takes just a few seconds, can contribute to reducing sintering time and, hence, manufacturing costs.

\section{Conclusions}

From consideration of two different silver nanoparticle inks on two different substrates we see that the effectiveness of the sintering approach is highly dependent on the matched properties of both ink and substrate material. An overview of the sintering results obtained are shown in Table 2, demonstrating that the applied sintering technology has to be carefully chosen for each ink/substrate combination in order to obtain optimal electrical properties of the printed patterns.

The comparison of the different paper substrates revealed that PEL paper with its micro porous inorganic ink receiving layer is more stable under all applied sintering conditions, whereas tattoo paper is more heat sensitive due to the thin polymer receiving layer, which does not tolerate temperatures above $100{ }^{\circ} \mathrm{C}$ for an extended amount of time. Therefore, global or selective heating often resulted in damaged substrate and sintered pattern and sintering temperatures and conditions have to be chosen as mild as possible.

Optimized conditions for each ink/paper/sintering combination were subsequently used for the manufacturing of FSS and RFID devices. This work demonstrates that single layer FSS and RFID conductive features can be produced on paper substrates via inkjet printing in combination with thermal and alternative sintering techniques compatible with R2R manufacturing. The performance of the FSS arrays meets reallife requirements for application in the field of wireless communication. We have furthermore demonstrated that the performance of the FSS presented here can be improved by adopting a multilayer printing approach by increasing the amount of deposited material. Triple layer FSS show transmission responses that are superior to previously reported inkjet printed FSS on PEN foil and comparable to copper etched according to the same design (Table S1 $\dagger$ ). Furthermore, it was found that on-skin RFID tags manufactured via inkjet printing and using plasma and IPL sintering methods show superior electrical and, hence, greater read distance performance compared to RFID tags previously produced by inkjet manufacturing. Although processing conditions have to be chosen carefully for the heat sensitive tattoo paper, the successful manufacturing of both, FSS and RFID tags on such a transferable substrate demonstrates the possibility of applying such devices on different surfaces such as walls, windows or onskin.

For the fabrication of paper based FSS arrays and RFID antennas, inkjet printing is a promising tool since it allows simple design alteration and adaption of layer thicknesses in order to tailor the properties of electromagnetically active structures to specific applications. Furthermore, recent developments in alternative sintering approaches show the potential to be used as fast and substrate friendly sintering technique allowing high throughput production in the near future. ${ }^{9,26,28,34}$

\section{Acknowledgements}

The authors thank Matthias Hartlieb and Stefan Hölzer from the Friedrich Schiller University Jena for assistance with the plasma sintering experiments. Veronica Sanchez-Romaguera, Daniel Tate Badredin Turki and Dumtoochukwu Oyeka would like to thank EPSRC for funding (EP/J000825/1 and EP/ K03099X/1). Authors would like to thank Prof. Madgassi for kindly providing Ink B. Veronica Sanchez-Romaguera and Sebastian Wünscher contributed equally to this work.

\section{References}

1 J. A. Arnaud and J. T. Ruscio, Electron. Lett., 1973, 9, 589-590. 
2 L. E. Comtesse, R. J. Langley, E. A. Parker and J. C. Vardaxouglou, in Proc. 17th European Mircowave Conf., Rome, 1987, vol. 9, pp. 2008-2013.

3 A. H. Wong, M. J. Neve and K. W. Sowerby, in Proc. IEEE Antenn. Propag. Soc. Int. Symp., Washington, CA, USA, 2005, vol. 1A, pp. 779-802.

4 E. A. Parker, J. B. Robertson, B. Sanz-Izquierdo and J. C. Batchelor, Electron. Lett., 2008, 44, 394-395.

5 J. C. Batchelor, E. A. Parker, J. A. Miller, V. SanchezRomaguera and S. G. Yeates, Electron. Lett., 2009, 45, 78.

6 W. G. Whittow, Y. Li, R. Torah, K. Yang, S. Beeby and J. Tudor, Electron. Lett., 2014, 50, 916-917.

7 S. Kim, B. Cook, T. R. Le, J. Cooper, H. Lee, V. Lakafosis, R. Vyas, R. Moro, M. Bozzi, A. Georgiadis, A. Collado and M. M. Tentzeris, IET Microwaves, Antennas and Propagation, 2013, 7, 858-868.

8 V. Sanchez-Romaguera, M. A. Ziai, D. Oyeka, S. Barbosa, J. S. R. Wheeler, J. C. Batchelor, E. A. Parker and S. G. Yeates, J. Mater. Chem. C, 2013, 1, 6395-6402.

9 S. Wünscher, R. Abbel, J. Perelaer and U. S. Schubert, J. Mater. Chem. C, 2014, 2, 10232-10261.

$10 \mathrm{~J}$. Perelaer, A. W. M. de Laat, C. E. Hendriks and U. S. Schubert, J. Mater. Chem., 2008, 18, 3209-3215.

11 S. Magdassi, M. Grouchko, O. Berezin and A. Kamyshny, ACS Nano, 2010, 4, 1943-1948.

12 S. M. Bidoki, D. M. Lewis, M. Clark, A. Vakorov, P. A. Millner and D. McGorman, J. Micromech. Microeng., 2007, 17, 967974.

13 M. L. Allen, M. Aronniemi, T. Mattila, A. Alastalo, K. Ojanperä, M. Suhonen and H. Seppä, Nanotechnology, 2008, 19, 175201.

14 I. Reinhold, C. E. Hendriks, R. Eckardt, J. M. Kranenburg, J. Perelaer, R. R. Baumann and U. S. Schubert, J. Mater. Chem., 2009, 19, 3384-3388.

15 J. Perelaer, R. Abbel, S. Wünscher, R. Jani, T. van Lammeren and U. S. Schubert, Adv. Mater., 2012, 24, 2620-2625.

16 J. Perelaer, B.-J. de Gans and U. S. Schubert, Adv. Mater., 2006, 18, 2101-2104.

17 D. Tobjörk, H. Aarnio, P. Pulkkinen, R. Bollström, A. Määttänen, P. Ihalainen, T. Mäkelä, J. Peltonen, M. Toivakka, H. Tenhu and R. Österbacka, Thin Solid Films, 2012, 520, 2949-2955.

18 T. Kumpulainen, J. Pekkanen, J. Valkama, J. Laakso, R. Tuokko and M. Mäntysalo, Opt. Laser Technol., 2011, 43, 570-576.

19 H.-S. Kim, S. R. Dhage, D.-E. Shim and H. T. Hahn, Appl. Phys. A, 2009, 97, 791-798.
20 R. Abbel, T. van Lammeren, R. Hendriks, J. Ploegmakers, E. J. Rubingh, E. R. Meinders and W. A. Groen, $M R S$ Commun., 2012, 2, 145-150.

21 Y. Amin, Q. Chen, L. R. Zheng and H. Tenhunen, Prog. Electromagn. Res., 2012, 130, 241-256.

22 N. Thi Thi, M. Nogi and K. Suganuma, J. Mater. Chem. C, 2013, 1, 5235-5243.

23 T. Öhlund, J. Örtegren, S. Forsberg and H.-E. Nilsson, Appl. Surf. Sci., 2012, 259, 731739.

24 L. Xie, M. Mäntysalo, A. López Cabezas, Y. Feng, F. Jonsson and L.-R. Zheng, Mater. Lett., 2012, 88, 68-72.

25 J. Niittynen, R. Abbel, M. Mäntysalo, J. Perelaer, U. S. Schubert and D. Lupo, Thin Solid Films, 2014, 556, 452-459.

26 A. Kamyshny and S. Magdassi, Small, 2014, 10, 3515-3535.

27 R. Abbel, T. van Lammeren, R. Hendriks, J. Ploegmakers, E. J. Rubingh, E. R. Meinders and W. A. Groen, $M R S$ Commun., 2012, 2, 145-150.

28 R. Abbel, P. Teunissen, E. Rubingh, T. Van Lammeren, R. Cauchois, M. Everaars, J. Valeton, S. van de Geijn and P. Groen, Transl. Mater. Res., 2014, 1, 015002.

29 M. Turki, E. A. Parker, J. C. Batchelor, M. A. Ziai, V. SanchezRomaguera and S. G. Yeates, Electron. Lett., 2013, 49, 10541055.

$30 \mathrm{http} / /$ www.printedelectronics.co.uk/Information\%20Sheet\% 20for\%20PEL\%20Nano\%20P60.pdf.

31 L. Goehring, R. Conroy, A. Akhter, W. J. Clegg and A. F. Routh, Soft Matter, 2010, 6, 3562-3567.

32 J. F. Salmerón, F. Molina-Lopez, D. Briand, J. J. Ruan, A. Rivadeneyra, M. A. Carvajal, L. F. Capitan Vallvey, N. F. Derooij and A. J. Palma, J. Electron. Mater., 2014, 43, 604-617.

33 K. C. Yung, X. Gu, C. P. Lee and H. S. Choy, J. Mater. Process. Technol., 2010, 210, 2268-2272.

34 M. Allen, J. Leppäniemi, M. Vilkman, A. Alastalo and T. Mattila, Nanotechnology, 2010, 21, 475204.

35 S. Wünscher, B. Seise, D. Pretzel, S. Pollok, J. Perelaer, K. Weber, J. Popp and U. S. Schubert, Lab Chip, 2014, 14, 392-401.

36 M. Frenkel, in The Chemistry of Inkjet Inks, ed. S. Magdassi, World ScientificPublishing Co. Pte. Ltd., Singapore, 2010, pp. 73-97.

37 K. A. Schroder, in Proc. NSIT Int. Nanotech. Conf., 2011, p. 220.

38 M. J. Guillot, S. C. McCool and K. A. Schroder, in Proc. 12th Int. Conf. Nucl. Engin.IMECE12 87674, 2012, vol. 7, pp. 19-27.

39 M. A. Ziai and J. C. Batchelor, IEEE Trans. Antennas Propag., 2011, 59, 3565-3571. 